DR ANDRIJA MATETIC (Orcid ID : 0000-0001-9272-6906)

DR MOHAMED OSAMA MOHAMED (Orcid ID : 0000-0002-9678-5222)

Article type : Original Paper

\title{
Trends, Management and Outcomes of Acute Myocardial Infarction in Chronic Liver Disease
}

Short title: AMI outcomes in liver disease patients

Andrija Matetic, $\mathrm{MD}^{\mathrm{a}, \mathrm{b}^{*}}$, Tahmeed Contractor, $\mathrm{MD}^{\mathrm{c} *}$, Mohamed O. Mohamed, MRCP(UK) ${ }^{\mathrm{d}, \mathrm{e}}$, Rahul Bhardwaj, MD ${ }^{\mathrm{c}}$, Ashish Aneja ${ }^{\mathrm{f}}$, Phyo K. Myint, MD, Mina O. Rakoski, MD MSc ${ }^{\mathrm{h}}$, Shelley Zieroth ${ }^{\mathrm{i}}$, Timir K. Paul, MD, PhDj, Mamas A. Mamas, DPhil ${ }^{\mathrm{d}, \mathrm{e}}$

*Joint first authors; contributed to manuscript equally

(a) Department of Cardiology, University Hospital of Split, Split, Croatia

(b) Department of Pathophysiology, University of Split School of Medicine, Split, Croatia

(c) Department of Cardiology, Loma Linda University, Loma Linda, CA, USA

(d) Keele Cardiovascular Research Group, Centre for Prognosis Research, Institutes of Applied Clinical Science and Primary Care and Health Sciences, Keele University, UK

(e) Department of Cardiology, Royal Stoke University Hospital, Stoke-on-Trent, UK

(f) MetroHealth Heart and Vascular, Case Western Reserve University, Cleveland, OH, USA

(g) Institute of Applied Health Sciences, University of Aberdeen

(h) Department of Gastroenterology and Hepatology, Loma Linda University, Loma Linda, CA, USA

(i) Section of Cardiology, University of Manitoba, Winnipeg, Canada

(j) East Tennessee State University, TN, USA

This article has been accepted for publication and undergone full peer review but has not been through the copyediting, typesetting, pagination and proofreading process, which may lead to differences between this version and the Version of Record. Please cite this article as doi: $\underline{10.1111 / \text { ijcp. } 13841}$

This article is protected by copyright. All rights reserved 
Disclosure: All authors disclose potential conflicts of interests.

\section{Correspondence to:}

Mamas A. Mamas

Professor of Cardiology

Keele Cardiovascular Research Group,

Centre for Prognosis Research,

Institute for Primary Care and Health Sciences, Keele University, UK

mamasmamas1@yahoo.co.uk

\section{Declarations of interest: None}

Word count (manuscript text): 3,450

\section{Abstract:}

Aims: There is limited data on the management and outcomes of chronic liver disease (CLD) patients presenting with acute myocardial infarction (AMI), particularly according to the subtype of CLD.

Methods: Using the Nationwide Inpatient Sample (2004-2015), we examined outcomes of AMI patients stratified by severity and sub-types of CLD. Multivariable logistic regression was performed to assess the adjusted odds ratios (aOR) of receipt of invasive management and adverse outcomes in CLD groups compared with no-CLD.

Results: Out of 7,024,723 AMI admissions, 54,283 (0.8\%) had a CLD diagnosis. CLD patients were less likely to undergo coronary angiography (CA) and percutaneous coronary intervention (PCI) (aOR $0.62,95 \%$ CI $0.60-0.63$ and $0.59,95 \% \mathrm{CI} 0.58-0.60$, respectively), and had increased odds of adverse outcomes including major adverse cardiovascular and cerebrovascular events $(1.19,95 \% \mathrm{CI} 1.15$ $1.23)$, mortality $(1.30,95 \% \mathrm{CI} 1.25-1.34)$ and major bleeding (1.74, 95\% CI 1.67-1.81). In comparison to the non-severe CLD sub-groups, patients with all forms of severe CLD had the lower utilization of 
CA and PCI $(\mathrm{p}<0.05)$. Amongst severe CLD patients, those with alcohol-related liver disease (ALD) had the lowest utilization of CA and PCI; patients with ALD and other CLD (OCLD) had more adverse outcomes than the viral hepatitis sub-group $(\mathrm{p}<0.05)$.

Conclusions: CLD patients presenting with AMI are less likely to receive invasive management and are associated with worse clinical outcomes. Further differences are observed depending on the type as well as severity of CLD, with the worst management and clinical outcomes observed in those with severe ALD and OCLD.

Key words: acute myocardial infarction; chronic liver disease; in-hospital outcomes.

What's known? There is limited data on management strategies and clinical outcomes of CLD patients when presenting with AMI from a national perspective. There have been only small studies on the invasive management and outcomes in a subset of such patients (e.g. ST-elevation myocardial infarction (STEMI) or AMI patients with cirrhosis), ${ }^{1,2}$ and these outcomes have not been systematically evaluated across CLD sub-groups.

What's new? This is the first study to examine the prevalence, management strategy and clinical outcomes of CLD patients hospitalized for AMI from a nationwide perspective. This article shows that patients with CLD are significantly less likely to receive invasive management for AMI compared to those without CLD, and are overall at a significantly increased risk of adverse outcomes.

This article is protected by copyright. All rights reserved 


\section{Introduction:}

Chronic liver disease (CLD) is a major cause of morbidity and mortality worldwide. ${ }^{3,4}$ The most common causes of CLD are chronic viral hepatitis (hepatitis B and C), alcohol-related liver disease (ALD), and non-alcoholic fatty liver disease (NAFLD). With continued insult, the early stages of CLD can progress with worsening fibrosis, leading to cirrhosis and end-stage liver disease. Despite the stable prevalence of chronic viral hepatitis and ALD, the overall burden of CLD continues to increase, primarily due to a rise in the incidence of NAFLD, which is largely attributed to an increase in obesity and diabetes mellitus. ${ }^{3-5}$ Coronary artery disease (CAD) is common amongst patients with CLD, with a $37 \%$ prevalence in liver transplant recipients. ${ }^{6-8}$ CLD patients presenting with acute myocardial infarction (AMI), pose a therapeutic dilemma for cardiologists. While their risk of cardiovascular mortality is at least as high as patients without CLD, they are at an inherent risk of major bleeding complications due to risk factors such as anemia, thrombocytopenia, decreased clotting factor synthesis, increased fibrinolysis and renal impairment. ${ }^{9-12}$ They may be less likely to receive guideline-recommended management in the form of percutaneous coronary intervention (PCI) and dual-antiplatelet therapy (DAPT) due to these increased risks. ${ }^{13-16}$ There is limited data on management strategies and clinical outcomes for this high-risk group when presenting with AMI from a national perspective. While there have been small studies on the utilization of invasive therapies and outcomes in a subset of such patients (e.g. ST-elevation myocardial infarction (STEMI) or AMI patients with cirrhosis), ${ }^{1,2}$ these outcomes have not been systematically evaluated across CLD subgroups. Using the National Inpatient Sample (NIS), we examined the prevalence of CLD amongst patients presenting with AMI over a 12-year period, and evaluated differences in their receipt of invasive management and subsequent clinical outcomes, compared to patients without CLD, stratified by CLD severity as well as subtype.

\section{Methods}

The NIS is the largest publicly available all-payer database of hospitalized patients in the United States and is sponsored by the Agency for Healthcare Research and Quality (AHRQ) which administers the Healthcare Cost and Utilization Project (HCUP). ${ }^{17}$ It includes anonymized data on

This article is protected by copyright. All rights reserved 
primary and secondary discharge diagnoses and procedures from $>7$ million hospitalizations annually. Further information on the design and validation of NIS dataset is provided in Appendix A.

All hospitalized adults ( $\geq 18$ years) with a principal discharge diagnosis of AMI between January 2004 and September 2015 were included. International Classification of Diseases, Ninth revision (ICD-9) and Clinical Classification Software (CCS) codes were used to identify patient comorbidities, procedures and clinical outcomes (Table S1). Additional comorbidities were identified using the existing 29 AHRQ Elixhauser comorbidity measures. Patient characteristics and clinical outcomes were stratified according to the presence or absence of CLD. Based on previous findings of different CLD etiologies influencing outcomes ${ }^{18}$ CLD patients were stratified, into 3 groups: chronic viral hepatitis, ALD and "other CLD“(OCLD) diagnoses (Table S1). To further assess differences in outcomes based on severity of CLD, groups were stratified into severe and non-severe categories, based on the presence of any of the following: portal hypertension, hepatic encephalopathy, hepatorenal syndrome and thrombocytopenia and coagulopathy. Cases excluded due to missing data for the variables Hospital bedsize and Hospital location/teaching status, and the presence of liver transplant represented $0.4 \%(n=28,752)$ of the original dataset (Figure S1). Before the exclusion of missing data, the dataset has been tested using Little's MCAR test which showed completely random missingness pattern [Chi-square $=1.985, \mathrm{DF}=2, \mathrm{p}=0.371]$. These variables were kept in the analysis to allow for better understanding of the results and proper adjustment in the multivariable model.

The main outcome was to compare the receipt of invasive management for AMI, in the form of coronary angiography (CA), percutaneous coronary intervention (PCI) and coronary artery bypass grafting (CABG), and subsequent in-hospital clinical outcomes in patients with and without CLD. Inhospital complications included major adverse cardiovascular and cerebrovascular events (MACCE), all-cause mortality, major bleeding, cardiac complications and acute stroke. MACCE was defined as a composite of all-cause mortality, acute stroke/transient ischemic attack (TIA) and cardiac complications. Cardiac complications included hemopericardium, cardiac tamponade, coronary dissection and any pericardiocentesis procedure. Major bleeding encompassed subarachnoid hemorrhage, intracerebral hemorrhage, intracranial hemorrhage, gastrointestinal hemorrhage, epistaxis and hemoptysis. All outcomes were based on in-hospital events/procedures irrespectively of the length of stay. However, almost all hospitalizations were shorter than 30 days $(99.5 \%)$.

This article is protected by copyright. All rights reserved 
We assessed the normality of data distribution by the Kolmogorov-Smirnov test. Data were expressed as median (interquartile range) for continuous non-parametric data and as numbers (percentages) for categorical data. Quantitative non-parametric data have been analysed with the Mann-Whitney U test and Kruskal-Wallis test, while the Chi-square test was used for the comparison of categorical variables between the study groups. Analyses were weighted by the provided discharge weights to allow estimation of national averages. All reported data were based on the weighted analyses as advised by HCUP. Multivariable logistic regression analysis was performed to determine adjusted odds ratios (aOR) of invasive management and adverse outcomes. We have adjusted for all baseline covariates that are included in the NIS, which we found both clinically meaningful and directly related to in-hospital outcomes. Further information on the statistical analysis and variables controlled for is provided on Appendix B. Statistical significance was defined at a level of $\mathrm{p}<0.05$. SPSS 25 software (IBM Corp, Armonk, NY) was used for statistical analysis.

\section{Results}

After excluding patients due to missing data (Figure S1), a total of 1,458,009 records of AMI between 2004 and September 2015 were identified, which corresponded to 7,024,723 hospitalizations. There were $6,970,440$ patients $(99.2 \%)$ in the no-CLD group and 54,283 patients $(0.8 \%)$ in the CLD group. Figure 1 displays the trends of CLD rates in AMI hospitalizations. The rate of CLD amongst patients hospitalized for AMI has shown a steady increase and more than doubled from $2004(0.5 \%)$ to $2015(1.1 \%)(\mathrm{p}<0.001$ for trend). Figure 2 shows the proportion of patients in the different subgroups of CLD; other causes accounted for most of these patients $(0.4 \%)$, followed by chronic hepatitis $(0.3 \%)$ and $\operatorname{ALD}(0.1 \%)$.

Demographics and comorbid conditions of the 2 groups are displayed in Table 1. Patients in the CLD group were more likely to be younger (median age 62 years in CLD group, 68 years in noCLD group, $\mathrm{p}<0.001)$, male $(65.8 \%$ in CLD group; $60.3 \%$ in no-CLD group, $\mathrm{p}<0.001$ ), and have a higher prevalence of medical comorbidities including anemia, coagulopathy, chronic pulmonary disease, diabetes mellitus, drug abuse, fluid and electrolyte abnormalities, and renal failure $(\mathrm{p}<0.001)$.

Patients without CLD had a higher percentage of dyslipidemia and were more likely to present with STEMI $(\mathrm{p}<0.001)$.

This article is protected by copyright. All rights reserved 
Management strategies utilized in the groups are shown in Figure 3A and Table 2. Patients with CLD had lower rates of CA $(57.5 \%$ versus $65.0 \%, \mathrm{p}<0.001)$ and PCI $(31.9 \%$ versus $43.3 \%$, $\mathrm{p}<0.001)$ as compared with no-CLD. The CLD group was less likely to undergo CA and PCI (aOR 0.62 ; $95 \%$ CI 0.60 to 0.63 , and aOR $0.59 ; 95 \%$ CI 0.58 to 0.60 ), respectively (Figure 4 and Table 3 ). Within the CLD sub-groups, all sub-groups were less likely to receive CA or PCI $(\mathrm{p}<0.05)$ (Figure 5 and Table 3).

When comparing within sub-groups of CLD based on severity, severe form of all sub-groups had lower odds of CA and PCI compared to their non-severe counterparts $(\mathrm{p}<0.05)$ (Table 3$)$. Amongst the severe sub-groups, patients with severe ALD had the lowest odds of CA and PCI, followed by patients with the severe form of OCLD; while the chronic viral hepatitis sub-group had the most favorable odds of undergoing CA and PCI amongst severe patients $(p<0.05)($ Table 3$)$.

A comparison of overall rates of in-hospital outcomes are displayed in Figure 3B and Table 2. The rates of MACCE (8.4\% in CLD group, $7.0 \%$ in no-CLD group, $\mathrm{p}<0.001$ ), all-cause mortality (7.3\% in CLD group, $5.7 \%$ in no-CLD group, $\mathrm{p}<0.001$ ), major bleeding (4.7\% in CLD group, $2.5 \%$ in no-CLD group, $\mathrm{p}<0.001$ ) were higher in patients with CLD, while the rates of cardiac complications were similar between the 2 groups $(0.1 \%, \mathrm{p}=0.056)$.

Figure 6 shows a comparison of inpatient outcomes between the various subgroups of CLD, according to severity. Amongst the CLD subtypes, patients with severe forms of OCLD and ALD had statistically significantly higher rates of MACCE $(17.4 \%$ vs. $7.4 \%, p<0.001$ and $15.4 \%$ vs. $12.3 \%$, $\mathrm{p}=0.001)$, mortality $(16.1 \%$ vs. $6.5 \%, \mathrm{p}<0.001$ and $14.2 \%$ vs. $11.1 \%, \mathrm{p}<0.001)$ and major bleeding (7.1\% vs. $3.9 \%, p<0.001$ and $11.4 \%$ vs. $7.2 \%, p<0.001)$ compared to their non-severe counterparts, even though absolute differences in the ALD sub-group were less obvious. Severe chronic viral hepatitis sub-group showed higher rates of major bleeding $(3.9 \%$ vs. $1.8 \%, \mathrm{p}<0.001)$ in comparison to their non-severe equivalents, while differences in MACCE and mortality were attenuated (7.9\% vs. $6.3 \%, p=0.026$ and $6.0 \%$ vs. $5.0 \%, p=0.128$, respectively).

The aORs of adverse events in the CLD group and the different CLD sub-groups are displayed in Table 3 and Figures 4-5. Patients with any CLD had a higher odds of MACCE (aOR 1.19, 95\% CI 
1.15-1.23), all-cause mortality (aOR 1.30, 95\% CI 1.25-1.34) and major bleeding (aOR 1.74, 95\% CI 1.67-1.81). However, the odds of stroke was lower in patients with CLD (aOR 0.83, CI 0.77-0.89).

When evaluating CLD sub-groups, most demonstrated higher odds of MACCE, mortality and major bleeding (Table 3). Compared to the no-CLD group, the odds of stroke varied amongst the CLD sub-groups with lower odds in non-severe OCLD (aOR 0.78, 95\% CI 0.69 to 0.87), severe OCLD (aOR 0.62, 95\% CI 0.40 to 0.97) and severe ALD (aOR 0.59, 95\% CI 0.37 to 0.92), but not statistically significantly different in other sub-groups ( $>0.05$ ) (Figure 5 and Table 3 ).

When comparing the CLD sub-groups based on severity, severe OCLD patients had higher odds of MACCE, mortality and major bleeding compared to their non-severe counterparts; severe ALD patients had only higher odds of major bleeding; while patients with severe form of chronic viral hepatitis had lower odds of MACCE and mortality compared to the non-severe subgroup $(p<0.05)$ (Table 3). When comparing the severe sub-groups of CLD, patients with OCLD and ALD had similarly higher odds of MACCE, mortality and major bleeding $(\mathrm{p}<0.05)$ (Table 3$)$.

Following adjustement for differences in baseline characteristics amongst patients who underwent PCI, aORs indicated that the odds were significantly higher for MACCE (aOR 1.12, 95\% CI 1.04 to 1.21 ), mortality (aOR $1.17,95 \%$ CI 1.08 to 1.28 ) and major bleeding (aOR $1.65,95 \% \mathrm{CI}$ 1.51 to 1.80 ; Table S2) in patients with CLD.

The trends of adverse events are listed in Table S3 and further illustrated in Figure S2. Overall, there was a trend for a decline in the odds of MACCE (aOR 1.42 in 2004, 1.05 in 2015), allcause mortality (aOR 1.45 in 2004, 1.13 in 2015) and stroke (aOR 1.36 in 2004, 0.82 in 2015), and an increase in major bleeding (aOR 1.90 in 2004, 2.16 in 2015), in patients with CLD compared to patients without CLD ( $<<0.001$ for trend).

\section{Discussion:}

This is the first study to examine the prevalence, management strategy and clinical outcomes of CLD patients hospitalized for AMI from a nationwide perspective. This study shows that the prevalence of CLD among patients with AMI has doubled between 2004 and 2015. Patients with CLD were significantly less likely to receive invasive management for AMI compared to those without 
CLD, and were overall at a significantly increased risk of all-cause mortality as well as major complications including major bleeding. Additionally, we show that the differences in management strategies and outcomes of patients with CLD depend on the severity as well as the etiology. In comparison to their non-severe counterparts, severe CLD sub-groups had lower odds of CA and PCI, while the severe form of OCLD also showed higher MACCE, mortality and major bleeding. In the severe disease patients, sub-group with ALD had the lowest odds of CA and PCI, while patients with ALD and OCLD had similarly higher risks of MACCE, mortality and major bleeding.

The trends of CLD rates in patients presenting with AMI have not been systematically evaluated. This is the first, large scale evaluation demonstrating that the rates have steadily increased and doubled from $0.5 \%$ in 2004 to $1.1 \%$ in 2015 . The rates of CLD in patients presenting with AMI in our study was similar to a prior, smaller analysis of the NIS ( $\sim 10 \%$ sample size of the current study), that looked at cirrhosis alone and demonstrated a rate of approximately $0.5 \% .{ }^{1}$ Based on epidemiological data, the increase in rate of CLD seen in our study over the years is likely related to an associated increase in the prevalence of NAFLD in the population. ${ }^{5}$

Patients with CLD that have AMI can pose a challenge to treating clinicians when considering invasive management strategies. A higher rate of the hematological derangements including anemia and coagulopathy can make the use of peri-procedural anticoagulation difficult. Additionally, these patients with a higher rate of renal dysfunction have an increased risk of developing contrast-induced nephropathy when undergoing invasive management. Expectedly, patients with cirrhosis and endstage liver disease who undergo PCI and CABG have a higher risk of complications as well as mortality. ${ }^{14-16,19,20}$ Similar to the findings seen in cirrhosis, patients with CLD have many of these comorbidities and thus are also at an increased risk of complications. This can lead to a 'risk-aversion' tendency among treating physicians leading to lower utilization of invasive procedures in patients with CLD. Previous studies that focused on patients with AMI and cirrhosis ${ }^{1,2}$ found that the utilization of invasive management is less in patients with cirrhosis. In this study, that included a larger group of patients with CLD, we show for the first time that the utilization of invasive management, including CA, PCI, CABG and thrombolytic therapy was lower in patients with CLD compared to those without CLD. Similar to findings in patients with end-stage liver diseae, we found

This article is protected by copyright. All rights reserved 
that CLD patients undergoing PCI had worse MACCE, mortality and major bleeding, when compared to patients without CLD.

This is the first study where differences in management between various sub-groups of CLD stratified by severity were analyzed. CA and PCI was used the least among patients with severe forms of CLD, with ALD showing the worst utilization as compared to other etiologies of CLD. There are many possible reasons for this. Stigmatization of patients that have alcohol dependence/abuse is a known phenomenon, ${ }^{21}$ and can lead to a lower utilization of invasive strategies. ALD patients can also present with withdrawal and delirium tremens, making the performance of invasive procedures difficult. Also, the rate of progression of liver disease with metabolic and hematological abnormalities, in general, is worse in patients with ALD compared to other CLD etiologies ${ }^{22}$ and may preclude providers from performing invasive procedures more so in this sub-group.

Prior smaller studies have shown worse outcomes including mortality in patients with cirrhosis and AMI, ${ }^{1,2}$ and this large analysis shows similar findings in the larger group of patients with CLD. The higher risk of adverse events in patients with CLD could be related to many of the abovementioned co-morbidities such as renal dysfunction and hematological derangements including thrombocytopenia, anemia and coagulopathy. Antiplatelet agents and statins, which form the mainstay of CAD and AMI therapy, are underutilized in patients with CLD. ${ }^{23}$ The utilization of invasive strategies has been associated with improved outcomes in this patient population, ${ }^{2}$ which could possibly also affect outcomes. Finally there may be an element of selection bias, whereby patients that are sicker are less likely to receive PCI and their worse outcomes may not be related to the lack of receipt of PCI, but rather to the disease process itself.

Differences in outcomes among the various subgroups of CLD presenting with AMI were also evaluated for the first time in this study. In comparison to patients with non-severe disease, subgroups with severe form of OCLD have shown higher odds of MACCE, mortality and major bleeding, while in the severe disease group, patients with ALD and OCLD did much worse than patients with CLD from chronic hepatitis. Interestingly, even though utilization of PCI amongst patients with severe disease was lowest in the ALD sub-group, patients with OCLD had similarly worse odds of mortality and MACCE as patients with ALD. Based on known epidemiological data, 
patients in the OCLD sub-group most commonly have NAFLD with a small proportion of having other relatively rare diseases (such as hemochromatosis, primary biliary cirrhosis, primary sclerosing cholangitis, Wilson's disease, alpha-1-antitrypsin deficiency). ${ }^{5}$ NAFLD patients likely have a similar metabolic as well as inflammatory milieu that is seen in patients with CAD. It is possible that the 'double-hit' of cardio-metabolic syndrome and severe hepatic dysfunction could contribute to bad outcomes in this group. The relative lack of cardiometabolic risk factors and slighty more appropriate utilization of invasive strategies in patients with chronic hepatitis could explain why outcomes in this group, while still worse than patients without CLD, were relatively better than other sub-groups of severe CLD.

Interestingly, most of the patients with chronic viral hepatitis showed severe features. Chronic viral hepatitis has been previously identified as the strongest predictor of liver cirrhosis, in comparison to other etiologies, ${ }^{24}$ and cirrhosis developing from hepatitis $\mathrm{C}$ infection was a leading cause of liver transplantation in the United States in 2015. ${ }^{25}$ Furthermore, it has been shown that large proportion of chronic viral hepatitis patients are being underdiagnosed which can contribute to late referral after the development of high-risk features. ${ }^{26}$ Finally, stable prevalence of chronic viral hepatitis over the studied period with ageing of the population and advancements in medical treatment could influence this subgroup distribution as well. ${ }^{27}$

The odds of major bleeding were higher in the overall group of patients with CLD, and highest amongst those with severe form of ALD and OCLD. This occurred despite a lower utilization of invasive management (CA and PCI) in these patients, indicating that these patients had a high propensity for bleeding. Amongst patients who did undergo PCI in the CLD group, there was also a higher risk of bleeding compared to those without CLD. This underscores the importance of carefully weighing risks versus benefits of both medical (especially anticoagulation/antiplatelet agents) and invasive strategies in this high-risk group (especially in the ALD and OCLD groups) presenting with AMI. Strategies such as radial access, use of less potent antithrombotic agents and third generation drug eluting stents that require short DAPT regimes would be considered when invasive strategy is planned. $28-31$

This article is protected by copyright. All rights reserved 
In contrast, the odds of ischemic stroke were reduced in the CLD group which was mainly driven by the OCLD subgroup. Similar findings have been previously described in another NIS based study, ${ }^{32}$ while a recent meta-analysis on cirrhotic patients revealed no difference in ischemic stroke risk. ${ }^{33}$ Several factors could have a role in the complex interplay of CLD and stroke. It is possible that coagulopathy associated with CLD stimulates an antithrombotic milieu by affecting the delicate balance between bleeding and thrombosis. Furthermore, the hyperdynamic circulation in CLD could have a protective effect on the formation of cerebral ischemic lesions. As previously shown, it is also possible that the etiology of CLD has an effect on the association of CLD and stroke. ${ }^{34}$ Different pathophysiology including predominantly impaired inflammatory cascade in chronic viral hepatitis and behavioral factors in ALD could lead to a diverse impact on stroke development in comparison to the OCLD patient group.

This study has several limitations that are inherent to the nature of analyzing data from an administrative dataset which is ICD code dependent and related to a specific hospitalization. There is always a possibility of misclassified, incomplete and omitted diagnoses or procedures. The specific etiologies of chronic hepatitis and other causes of CLD (eg. NAFLD) are unknown, given the limited ICD codes. Patients hospitalized with CLD are more likely to have a type 2 MI from etiologies such as sepsis, and associated vasodilatory shock. ${ }^{35,36}$ It is possible that some of the patients in the CLD group of this study were such patients, and hence, it is expected that the role of invasive management, as well as overall adverse outcomes would be different in this group. The type of medical management (antiplatelet agents, anticoagulation) also plays an important role in outcomes; and are not captured for individual patients from this dataset.

In conclusion, this is the first large study to delineate trends in the rates of CLD in patients presenting with AMI, that has steadily increased and doubled over a decade. The odds of invasive management are lower, and that of most adverse events are higher in patients with CLD who present with AMI. Patients with severe form of ALD had the worst utilization of CA and PCI. Furthermore, MACCE, mortality and bleeding were worse in patients with severe form of ALD and OCLD. Prospective evaluation of management strategies as well as longer term outcomes in the various subgroups of patients with CLD presenting with AMI are needed.

This article is protected by copyright. All rights reserved 


\section{Acknowledgments:}

Funding: None.

Declarations of interest: None.

Acknowledgments: None.

Author contributions: Andrija Matetic: Software, Data curation, Methodology, Formal analysis, Visualization, Writing- Reviewing and Editing; Tahmeed Contractor: Methodology, WritingOriginal draft preparation, Writing- Reviewing and Editing, Validation; Mohamed Mohamed: Conceptualization, Methodology, Data curation, Formal analysis, Writing- Reviewing and Editing; Rahul Bhardwaj: Validation, Writing- Reviewing and Editing; Ashish Aneja: Validation, WritingReviewing and Editing; Phyo K. Myint: Validation, Writing- Reviewing and Editing; Mina 0. Rakoski: Validation, Writing- Reviewing and Editing; Shelley Zieroth: Validation, WritingReviewing and Editing; Timir K. Paul: Validation, Writing- Reviewing and Editing; Mamas A. 
Mamas: Supervision, Conceptualization, Methodology, Resources, Project administration, Validation, Writing- Reviewing and Editing.

\section{References:}

1. Hillerson D, Ogunbayo GO, Salih M, et al. Outcomes and Characteristics of Myocardial Infarction in Patients With Cirrhosis. The Journal of invasive cardiology. 2019;31(7):E162E169.

2. Abougergi MS, Karagozian R, Grace ND, Saltzman JR, Qamar AA. ST elevation myocardial infarction mortality among patients with liver cirrhosis. Journal of clinical gastroenterology. 2015;49(9):778-783.

3. Sepanlou SG, Safiri S, Bisignano C, et al. The global, regional, and national burden of cirrhosis by cause in 195 countries and territories, 1990-2017: a systematic analysis for the Global Burden of Disease Study 2017. The Lancet Gastroenterology \& Hepatology. 2020;5(3):245-266.

4. Murray CJ, Abraham J, Ali MK, et al. The state of US health, 1990-2010: burden of diseases, injuries, and risk factors. Jama. 2013;310(6):591-606.

5. Moon AM, Singal AG, Tapper EB. Contemporary epidemiology of chronic liver disease and cirrhosis. Clinical Gastroenterology and Hepatology. 2019.

6. An J, Shim JH, Kim S-O, et al. Prevalence and prediction of coronary artery disease in patients with liver cirrhosis: a registry-based matched case-control study. Circulation. 2014;130(16):1353-1362.

This article is protected by copyright. All rights reserved 
7. Patel SS, Nabi E, Guzman L, et al. Coronary artery disease in decompensated patients undergoing liver transplantation evaluation. Liver transplantation. 2018;24(3):333-342.

8. Kalaitzakis E, Rosengren A, Skommevik T, Björnsson E. Coronary artery disease in patients with liver cirrhosis. Digestive diseases and sciences. 2010;55(2):467-475.

9. Hartleb M, Gutkowski K. Kidneys in chronic liver diseases. World journal of gastroenterology: WJG. 2012;18(24):3035.

10. Gonzalez-Casas R, Jones EA, Moreno-Otero R. Spectrum of anemia associated with chronic liver disease. World journal of gastroenterology: WJG. 2009;15(37):4653.

11. Afdhal N, McHutchison J, Brown R, et al. Thrombocytopenia associated with chronic liver disease. Journal of hepatology. 2008;48(6):1000-1007.

12. Tripodi A, Mannucci PM. The coagulopathy of chronic liver disease. New England Journal of Medicine. 2011;365(2):147-156.

13. Wu VC-C, Chen S-W, Chou A-H, et al. Dual antiplatelet therapy in patients with cirrhosis and acute myocardial infarction-A 13-year nationwide cohort study. PloS one. 2019;14(10).

14. Kolte D, Khera S, Sharma N, et al. Outcomes of percutaneous coronary intervention in patients with cirrhosis. Journal of the American College of Cardiology. 2014;63(12 Supplement):A1849.

15. Shaheen AAM, Kaplan GG, Hubbard JN, Myers RP. Morbidity and mortality following coronary artery bypass graft surgery in patients with cirrhosis: a population-based study. Liver International. 2009;29(8):1141-1151.

16. Lu DY, Steitieh D, Feldman DN, et al. Impact Of Cirrhosis On 90-Day Outcomes After Percutaneous Coronary Intervention (From A Nationwide Database). The American Journal of Cardiology. 2020.

17. Rockville M. HCUP National Inpatient Sample (NIS). Healthcare Cost and Utilization Project (HCUP). 2012.

18. Collaborators GBDC. The global, regional, and national burden of cirrhosis by cause in 195 countries and territories, 1990-2017: a systematic analysis for the Global Burden of Disease Study 2017. Lancet Gastroenterol Hepatol. 2020;5(3):245-266.

This article is protected by copyright. All rights reserved 
19. Singh V, Patel NJ, Rodriguez AP, et al. Percutaneous coronary intervention in patients with end-stage liver disease. The American journal of cardiology. 2016;117(11):1729-1734.

20. Sharma M, Yong C, Majure D, et al. Safety of cardiac catheterization in patients with endstage liver disease awaiting liver transplantation. The American journal of cardiology. 2009;103(5):742-746.

21. Ronzani TM, Higgins-Biddle J, Furtado EF. Stigmatization of alcohol and other drug users by primary care providers in Southeast Brazil. Social science \& medicine. 2009;69(7):1080-1084.

22. Toshikuni N, Tsutsumi M, Arisawa T. Clinical differences between alcoholic liver disease and nonalcoholic fatty liver disease. World Journal of Gastroenterology: WJG. 2014;20(26):8393.

23. Patel SS, Guzman LA, Lin FP, et al. Utilization of aspirin and statin in management of coronary artery disease in patients with cirrhosis undergoing liver transplant evaluation. Liver Transplantation. 2018;24(7):872-880.

24. Scaglione S, Kliethermes S, Cao G, et al. The Epidemiology of Cirrhosis in the United States: A Population-based Study. Journal of clinical gastroenterology. 2015;49(8):690-696.

25. Organization WH. Global hepatitis report 2017. 2017.

26. Yehia BR, Schranz AJ, Umscheid CA, Lo Re V, 3rd. The treatment cascade for chronic hepatitis $\mathrm{C}$ virus infection in the United States: a systematic review and meta-analysis. PLoS One. 2014;9(7):e101554-e101554.

27. Younossi ZM, Stepanova M, Afendy M, et al. Changes in the prevalence of the most common causes of chronic liver diseases in the United States from 1988 to 2008. Clinical gastroenterology and hepatology : the official clinical practice journal of the American Gastroenterological Association. 2011;9(6):524-530.e521; quiz e560.

28. Jacobs E, Singh V, Damluji A, et al. Safety of transradial cardiac catheterization in patients with end-stage liver disease. Catheterization and Cardiovascular Interventions. 2014;83(3):360-366.

29. Pillarisetti J, Patel P, Duthuluru S, et al. Cardiac catheterization in patients with end-stage liver disease: Safety and outcomes. Catheterization and Cardiovascular Interventions. 2011;77(1):45-48.

This article is protected by copyright. All rights reserved 
30. Urban P, Meredith IT, Abizaid A, et al. Polymer-free Drug-Coated Coronary Stents in Patients at High Bleeding Risk. The New England journal of medicine. 2015;373(21):2038-2047.

31. Windecker S, Latib A, Kedhi E, et al. Polymer-based or Polymer-free Stents in Patients at High Bleeding Risk. The New England journal of medicine. 2020;382(13):1208-1218.

32. Goyal A, Chatterjee K, Shah N, Singh S. Is cirrhosis associated with lower odds of ischemic stroke: A nationwide analysis? World J Hepatol. 2016;8(35):1564-1568.

33. Zheng K, Yoshida EM, Tacke F, Li Y, Guo X, Qi X. Risk of Stroke in Liver Cirrhosis: A Systematic Review and Meta-Analysis. Journal of clinical gastroenterology. 2020;54(1):96105.

34. Zhang X, Qi X, Yoshida EM, et al. Ischemic stroke in liver cirrhosis: epidemiology, risk factors, and in-hospital outcomes. European journal of gastroenterology \& hepatology. 2018;30(2):233-240.

35. Foreman MG, Mannino DM, Moss M. Cirrhosis as a risk factor for sepsis and death: analysis of the National Hospital Discharge Survey. Chest. 2003;124(3):1016-1020.

36. $\mathrm{Wu} \mathrm{AH}$. Increased troponin in patients with sepsis and septic shock: myocardial necrosis or reversible myocardial depression? In: Springer; 2001.

\section{Figure titles and legends:}

\section{Figure 1. Rate of CLD amongst AMI hospitalizations (2004-2015*).}

Legend: *2015 - 1st January through $30^{\text {th }}$ September only; AMI - acute myocardial infarction; CLD - chronic liver disease.

\section{Figure 2. Frequency of different CLD subtypes in AMI population.}

Legend: AMI - acute myocardial infarction; CLD - chronic liver disease.

Figure 3. Comparison between study groups: A. Invasive management; B. In-hospital outcomes.

Legend: CA - Coronary Angiography; CA - Coronary Artery Bypass Grafting; CLD - chronic liver disease; MACCE major adverse cardiovascular and cerebrovascular events (composite of mortality, cardiac complications and stroke); PCI

- Percutaneous Coronary Intervention. 


\section{Figure 4. Adjusted odds ratios (aOR) of outcomes in CLD group*.}

Legend: *reference is no CLD group; CLD - chronic liver disease; MACCE - major adverse cardiovascular and cerebrovascular events (composite of mortality, cardiac complications and stroke).

Figure 5. Adjusted odds ratios (aOR) of outcomes in different CLD subgroups*: A. Non-severe

\section{CLD; B. Severe CLD $\uparrow$.}

Legend: *reference is no CLD group; $\uparrow$ Presence of portal hypertension, hepatic encephalopathy, hepatorenal syndrome and thrombocytopenia and coagulopathy; CA - Coronary Angiography; CLD - chronic liver disease; MACCE - major adverse cardiovascular and cerebrovascular events (composite of mortality, cardiac complications and stroke); PCI Percutaneous Coronary Intervention.

\section{Figure 6. In-hospital outcomes of different CLD subtypes based on disease severity.}

Legend: *Presence of portal hypertension, hepatic encephalopathy, hepatorenal syndrome, thrombocytopenia and coagulopathy; CLD - chronic liver disease; MACCE - major adverse cardiovascular and cerebrovascular events (composite of mortality, cardiac complications and stroke). 
Table 1. Patient characteristics of study groups

\begin{tabular}{|c|c|c|c|}
\hline Variable/Group (\%) & $\begin{array}{l}\text { No CLD } \\
(99.2 \%)\end{array}$ & CLD $(0.8 \%)$ & p-value \\
\hline Number of hospitalizations & $6,970,440$ & 54,283 & \\
\hline Age (years), median (IQR) & $68(57,79)$ & $62(54,71)$ & $<0.001$ \\
\hline Males, \% & 60.3 & 65.8 & $<0.001$ \\
\hline STEMI, \% & 29.4 & 20.1 & $<0.001$ \\
\hline Weekend admission, \% & 26.0 & 26.2 & 0.255 \\
\hline Primary expected payer, \% & & & $<0.001$ \\
\hline Medicare & 57.4 & 51.6 & \\
\hline Medicaid & 6.0 & 14.0 & \\
\hline Private Insurance & 27.7 & 24.0 & \\
\hline Self-pay & 5.7 & 5.7 & \\
\hline No charge & 0.6 & 0.7 & \\
\hline Other & 2.7 & 4.1 & \\
\hline Median Household Income Percentile, \% & & & $<0.001$ \\
\hline $0-25^{\text {th }}$ & 28.6 & 31.2 & \\
\hline $26-50^{\text {th }}$ & 27.3 & 26.5 & \\
\hline $51-75^{\text {th }}$ & 23.8 & 22.4 & \\
\hline $76-100^{\text {th }}$ & 20.3 & 19.8 & \\
\hline Cardiogenic shock, \% & 5.0 & 5.4 & 0.001 \\
\hline Cardiac arrest, \% & 1.6 & 1.9 & $<0.001$ \\
\hline Ventricular tachycardia, \% & 2.6 & 2.7 & 0.033 \\
\hline Ventricular fibrillation, \% & 2.7 & 2.3 & $<0.001$ \\
\hline Coronary dissection, \% & 0.5 & 0.5 & 0.874 \\
\hline Cardiac tamponade, $\%$ & 0.1 & 0.0 & 0.024 \\
\hline Hemopericardium, \% & 0.0 & 0.0 & 0.329 \\
\hline Pericardiocentesis, \% & 0.0 & 0.0 & 0.996 \\
\hline \multicolumn{4}{|l|}{ Comorbidities, \% } \\
\hline $\mathbf{A F}$ & 16.6 & 16.6 & 0.854 \\
\hline
\end{tabular}

This article is protected by copyright. All rights reserved 


\begin{tabular}{|c|c|c|c|}
\hline Variable/Group (\%) & $\begin{array}{l}\text { No CLD } \\
(99.2 \%)\end{array}$ & CLD (0.8\%) & p-value \\
\hline Dyslipidaemia & 55.1 & 44.8 & $<0.001$ \\
\hline Thrombocytopenia & 2.3 & 12.3 & $<0.001$ \\
\hline Dementia & 1.7 & 0.8 & $<0.001$ \\
\hline Smoking history & 28.4 & 31.2 & $<0.001$ \\
\hline Previous AMI & 8.6 & 7.7 & $<0.001$ \\
\hline History of IHD & 76.9 & 69.6 & $<0.001$ \\
\hline Previous PCI & 9.7 & 7.3 & $<0.001$ \\
\hline Previous CABG & 6.1 & 4.3 & $<0.001$ \\
\hline Previous CVA & 3.1 & 2.4 & $<0.001$ \\
\hline Family history of CAD & 6.8 & 4.3 & $<0.001$ \\
\hline Deficiency anaemias & 14.7 & 23.0 & $<0.001$ \\
\hline Chronic blood loss anaemia & 1.1 & 2.4 & $<0.001$ \\
\hline Congestive heart failure & 0.9 & 0.9 & 0.971 \\
\hline Chronic pulmonary disease & 20.7 & 28.0 & $<0.001$ \\
\hline Coagulopathy & 4.3 & 21.0 & $<0.001$ \\
\hline Depression & 6.4 & 8.9 & $<0.001$ \\
\hline Diabetes & 28.2 & 33.6 & $<0.001$ \\
\hline Diabetes with complications & 6.1 & 9.8 & $<0.001$ \\
\hline Drug abuse & 2.0 & 9.3 & $<0.001$ \\
\hline Hypertension & 66.9 & 65.6 & $<0.001$ \\
\hline Hypothyroidism & 9.8 & 10.0 & 0.126 \\
\hline Lymphomas & 0.5 & 0.7 & $<0.001$ \\
\hline Fluid and electrolyte disturbances & 19.3 & 30.1 & $<0.001$ \\
\hline $\begin{array}{l}\text { Rheumatoid arthritis/collagen vascular } \\
\text { diseases }\end{array}$ & 2.2 & 2.6 & $<0.001$ \\
\hline Metastatic cancer & 0.9 & 1.1 & $<0.001$ \\
\hline Other neurological disorders & 5.8 & 6.7 & $<0.001$ \\
\hline Obesity & 12.0 & 15.8 & $<0.001$ \\
\hline
\end{tabular}

This article is protected by copyright. All rights reserved 


\begin{tabular}{lccc}
\hline Variable/Group (\%) & $\begin{array}{c}\text { No CLD } \\
(\mathbf{9 9 . 2 \% )}\end{array}$ & CLD (0.8\%) & p-value \\
\hline Paralysis & 1.6 & 1.4 & $<0.001$ \\
\hline Peripheral vascular disease & 10.9 & 12.2 & $<0.001$ \\
\hline Psychoses & 2.1 & 4.5 & $<0.001$ \\
\hline Pulmonary circulation disorder & 0.1 & 0.2 & $<0.001$ \\
\hline Renal failure (chronic) & 16.7 & 24.0 & $<0.001$ \\
\hline Solid tumour without metastases & 1.4 & 2.6 & $<0.001$ \\
\hline Ulcer disease & 0.0 & 0.1 & $<0.001$ \\
\hline Valvular heart disease & 0.2 & 0.3 & 0.001 \\
\hline AIDS & 0.1 & 1.1 & $<0.001$ \\
\hline Weight loss & 2.2 & 4.2 & $<0.001$ \\
\hline Hospital bed size, \% & & & $<0.001$ \\
\hline Small & 10.7 & 9.7 & \\
\hline Medium & 24.9 & 24.7 & \\
\hline Large & 64.5 & 65.6 & \\
\hline Hospital Region, \% & & & $<0.001$ \\
\hline Northeast & 19.2 & 18.0 & \\
\hline Midwest & 23.3 & 18.9 & \\
\hline South & 40.0 & 40.0 & \\
\hline West & 17.5 & 23.1 & \\
\hline Location/ Teaching status, \% & & & $<0.001$ \\
\hline Rural & 48.7 & 39.6 & \\
\hline Urban non-teaching & 52.4 & \\
\hline Urban- teaching & & & \\
\hline & & & \\
\hline
\end{tabular}

Legend: AF: atrial fibrillation; AIDS: acquired immunodeficiency syndrome; AMI: acute myocardial infarction; CABG: coronary artery bypass grafting; CAD: coronary artery disease; CLD: chronic liver disease; CVA: cerebrovascular accident; IABP: intra-aortic balloon pump; IHD: ischemic heart disease; IQR: interquartile range; PCI: percutaneous coronary intervention; STEMI: ST-elevation myocardial infarction.

This article is protected by copyright. All rights reserved 
Table 2. Unadjusted rates of in-hospital outcomes according to CLD and its subtypes

\begin{tabular}{|c|c|c|c|c|c|c|c|c|c|c|c|c|}
\hline \multirow[b]{2}{*}{ Variables } & \multirow{2}{*}{$\begin{array}{c}\text { No CLD } \\
\text { (N=6,970,440; } \\
\text { 99.4) }\end{array}$} & \multirow{2}{*}{$\begin{array}{c}\text { Total CLD } \\
(\mathrm{N}=54,283 \\
0.8 \%)\end{array}$} & \multirow[b]{2}{*}{ p-value* } & \multicolumn{3}{|c|}{$\begin{array}{l}\text { Chronic viral hepatitis } \\
\qquad(\mathrm{N}=17,683 ; 0.3 \%)\end{array}$} & \multicolumn{3}{|c|}{ 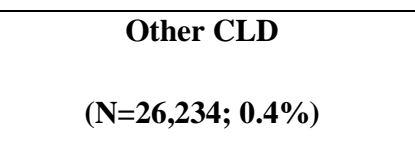 } & \multicolumn{3}{|c|}{$\begin{array}{l}\text { Alcohol-related CLD } \\
\qquad(\mathrm{N}=10,366 ; 0.1 \%)\end{array}$} \\
\hline & & & & $\begin{array}{l}\text { Non- } \\
\text { severe } \\
(7.4 \%)\end{array}$ & $\begin{array}{l}\text { Severe } \\
(92.6 \%)\end{array}$ & $\begin{array}{c}\text { p- } \\
\text { value } \dagger\end{array}$ & $\begin{array}{l}\text { Non- } \\
\text { severe } \\
(94.2 \%)\end{array}$ & $\begin{array}{l}\text { Severe } \\
(5.8 \%)\end{array}$ & $\begin{array}{c}\text { p- } \\
\text { value } \dagger\end{array}$ & $\begin{array}{c}\text { Non- } \\
\text { severe } \\
(84.2 \%)\end{array}$ & $\begin{array}{l}\text { Severe } \\
(\mathbf{1 5 . 8 \%})\end{array}$ & $\begin{array}{c}\text { p- } \\
\text { value } \uparrow\end{array}$ \\
\hline МАCCE, \% & 7.0 & 8.4 & $<0.001$ & 7.9 & 6.3 & 0.026 & 7.4 & 17.4 & $<0.001$ & 12.3 & 15.4 & 0.001 \\
\hline $\begin{array}{l}\text { All-cause mortality, } \\
\%\end{array}$ & 5.7 & 7.3 & $<0.001$ & 6.0 & 5.0 & 0.128 & 6.5 & 16.1 & $<0.001$ & 11.1 & 14.2 & $<0.001$ \\
\hline Major bleeding, \% & 2.5 & 4.7 & $<0.001$ & 1.8 & 3.9 & $<0.001$ & 3.9 & 7.1 & $<0.001$ & 7.2 & 11.4 & $<0.001$ \\
\hline $\begin{array}{c}\text { Cardiac } \\
\text { complications, \% }\end{array}$ & 0.1 & 0.1 & 0.056 & 0.0 & 0.0 & 0.573 & 0.1 & 0.3 & $<0.001$ & 0.1 & 0.0 & 0.289 \\
\hline $\begin{array}{r}\text { Postprocedural } \\
\text { haemorrhage, \% }\end{array}$ & 0.7 & 0.7 & 0.295 & 1.5 & 1.1 & 0.165 & 0.5 & 1.0 & 0.013 & 0.4 & 0.6 & 0.205 \\
\hline Stroke, \% & 1.5 & 1.4 & 0.026 & 1.9 & 1.4 & 0.161 & 1.2 & 1.3 & 0.731 & 1.7 & 1.2 & 0.092 \\
\hline $\mathrm{CA}, \%$ & 65.0 & 57.5 & $<0.001$ & 61.1 & 62.4 & 0.368 & 59.7 & 40.6 & $<0.001$ & 49.2 & 33.4 & $<0.001$ \\
\hline PCI, \% & 43.3 & 31.9 & $<0.001$ & 35.7 & 34.9 & 0.546 & 34.3 & 21.1 & $<0.001$ & 24.4 & 14.3 & $<0.001$ \\
\hline CABG, $\%$ & 8.9 & 8.6 & 0.020 & 6.9 & 10.0 & $<0.001$ & 8.6 & 6.1 & 0.001 & 7.0 & 5.5 & 0.024 \\
\hline Thrombolysis, \% & 1.4 & 1.0 & $<0.001$ & 0.8 & 1.2 & 0.271 & 1.0 & 1.3 & 0.260 & 0.9 & 0.6 & 0.132 \\
\hline $\begin{array}{l}\text { Use of IABP or } \\
\text { assist device, \% }\end{array}$ & 4.9 & 4.2 & $<0.001$ & 3.1 & 4.5 & 0.013 & 3.7 & 4.0 & 0.600 & 5.2 & 3.4 & 0.002 \\
\hline
\end{tabular}

This article is protected by copyright. All rights reserved 


\begin{tabular}{|c|c|c|c|c|c|c|c|c|c|c|c|c|}
\hline Length of stay & & & & & & & & & & & & \\
\hline $\begin{array}{c}\text { (days), median } \\
\text { (IQR) }\end{array}$ & $3(2,6)$ & $4(2,7)$ & $<0.001$ & $3(2,6)$ & $4(2,7)$ & 0.001 & $4(2,7)$ & $6(3,9)$ & $<0.001$ & $5(3,8)$ & $5(3,10)$ & $<0.001$ \\
\hline
\end{tabular}

*Comparison of no-CLD vs. total CLD group; †Comparison of subgroups based on severity within CLD subtype.

Legend: CA: coronary angiography; CABG: coronary artery bypass grafting; CLD: chronic liver disease; MACCE: major adverse cardiovascular and cerebrovascular events (composite of mortality, cardiac complications and stroke); PCI: percutaneous coronary intervention.

Table 3. Adjusted odds ratios (aOR) of adverse complications and invasive management in the CLD group and different CLD subgroups

This article is protected by copyright. All rights reserved 


\begin{tabular}{|c|c|c|c|c|c|c|c|}
\hline & $\begin{array}{l}\text { Total CLD } \\
\text { (N=54,283; }\end{array}$ & \multicolumn{2}{|c|}{$\begin{array}{l}\text { Chronic viral hepatitis } \\
\qquad(\mathrm{N}=17,683 ; 0.3 \%)\end{array}$} & \multicolumn{2}{|c|}{$\begin{array}{c}\text { Other CLD } \\
(\mathrm{N}=\mathbf{2 6 , 2 3 4 ; 0 . 4 \% )}\end{array}$} & \multicolumn{2}{|c|}{$\begin{array}{l}\text { Alcohol-related CLD } \\
\qquad(\mathrm{N}=10,366 ; 0.1 \%)\end{array}$} \\
\hline & OR $[95 \% \mathrm{CI}]$ & $\begin{array}{c}\text { Non-severe } \\
(7.4 \%)\end{array}$ & $\begin{array}{l}\text { Severe } \\
(92.6 \%)\end{array}$ & $\begin{array}{c}\text { Non-severe } \\
(94.2 \%)\end{array}$ & $\begin{array}{l}\text { Severe } \\
(5.8 \%)\end{array}$ & $\begin{array}{c}\text { Non-severe } \\
(84.2 \%)\end{array}$ & $\begin{array}{l}\text { Severe } \\
(15.8 \%)\end{array}$ \\
\hline & & OR [95\% CI] & OR $[95 \% \mathrm{CI}]$ & OR $[95 \% \mathrm{CI}]$ & OR $[95 \% \mathrm{CI}]$ & OR $[95 \%$ CI $]$ & OR $[95 \% \mathrm{CI}]$ \\
\hline MACCE & $1.19[1.15,1.23]$ & $1.26[1.02,1.55]$ & $1.04[0.98,1.11] \dagger \S$ & $1.10[1.04,1.15]$ & $2.08[1.81,2.38] \dagger+$ & $1.67[1.56,1.79]$ & $1.87[1.63,2.15] \ddagger$ \\
\hline Mortality & $1.30[1.25,1.34]$ & $1.20[0.95,1.52]$ & $1.05[0.98,1.13] \dagger \S$ & $1.20[1.14,1.26]$ & $2.39[2.07,2.76] \dagger+$ & $1.87[1.75,2.01]$ & $2.16[1.87,2.50] \ddagger$ \\
\hline $\begin{array}{c}\text { Major } \\
\text { bleeding }\end{array}$ & $1.74[1.67,1.81]$ & $0.75[0.50,1.12]$ & $1.55[1.43,1.68] \S$ & $1.52[1.43,1.62]$ & $2.11[1.73,2.58] \dagger+$ & $2.37[2.18,2.58]$ & $3.52[3.02,4.12]+t$ \\
\hline Stroke & $0.83[0.77,0.89]$ & $1.23[0.83,1.83]$ & $0.98[0.86,1.12]$ & $0.78[0.69,0.87]$ & $0.62[0.40,0.97]$ & $1.04[0.89,1.22]$ & $0.59[0.37,0.92] \dagger$ \\
\hline $\mathbf{C A}$ & $0.62[0.60,0.63]$ & $0.88[0.78,1.00]$ & $0.65[0.63,0.67] \dagger \S$ & $0.70[0.68,0.72]$ & $0.39[0.35,0.44] \dagger+$ & $0.48[0.46,0.50]$ & $0.25[0.23,0.28] \dagger \dagger \S$ \\
\hline PCI & $0.59[0.58,0.60]$ & $0.83[0.73,0.94]$ & $0.60[0.58,0.62] \dagger \S$ & $0.67[0.65,0.69]$ & $0.44[0.39,0.51] \dagger+$ & $0.44[0.42,0.46]$ & $0.25[0.21,0.28] \dagger \dagger \S$ \\
\hline
\end{tabular}

*Reference group is no chronic liver disease; $†$ Significant difference from non-severe CLD subtype; $¥$ Significant difference from severe chronic viral hepatitis subgroup; §Significant difference from severe 'other CLD' subgroup.

Legend: CA: coronary angiography; CI: confidence interval; CLD: chronic liver disease; MACCE: major adverse cardiovascular and cerebrovascular events (composite of mortality, cardiac complications and stroke); PCI: percutaneous coronary intervention.

This article is protected by copyright. All rights reserved 


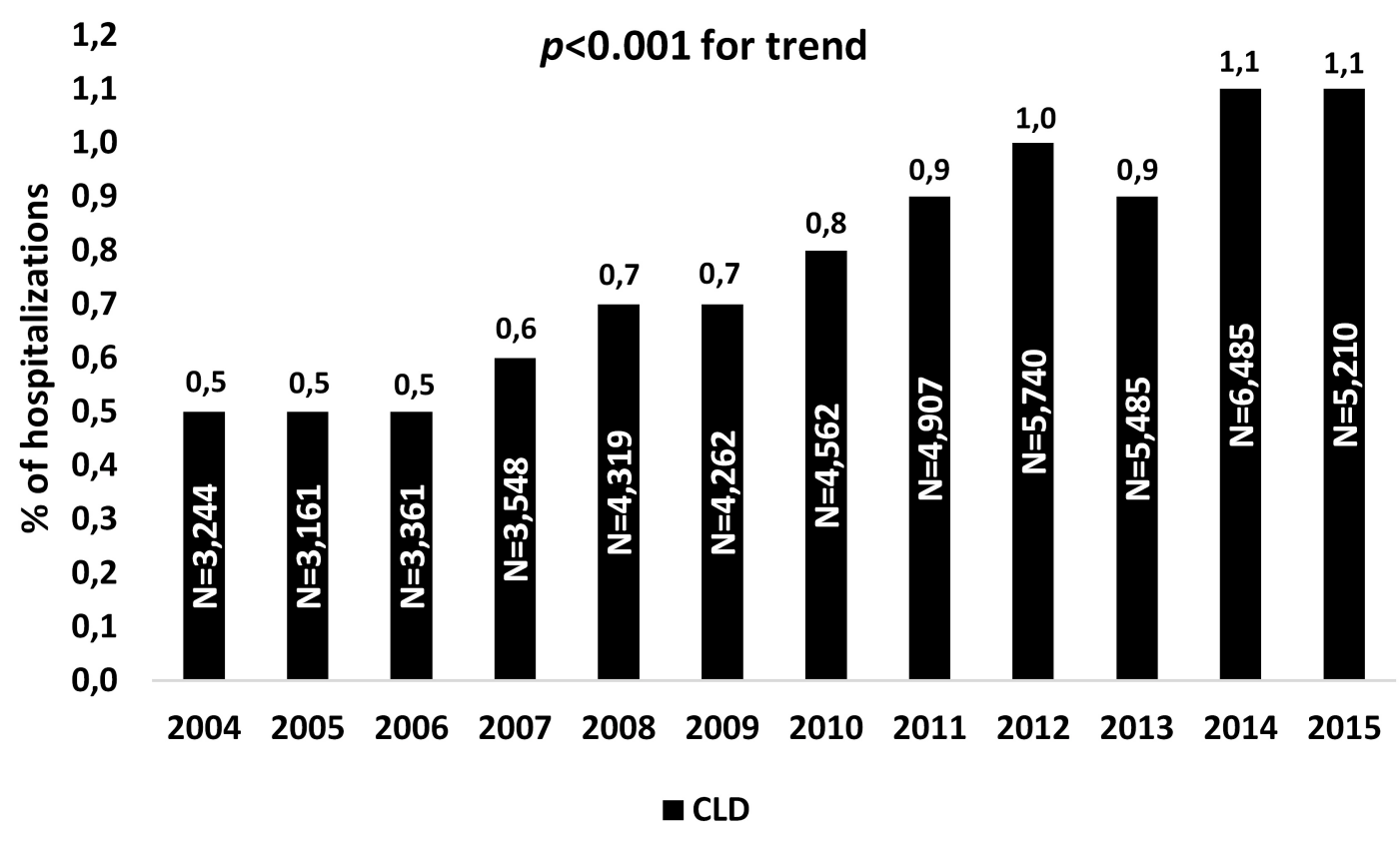

ijcp_13841_f1.jpg 


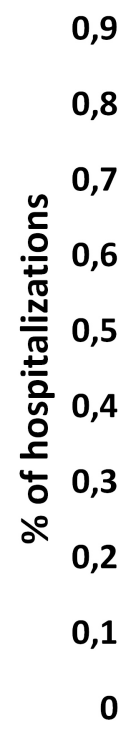

Chronic viral hepatitis

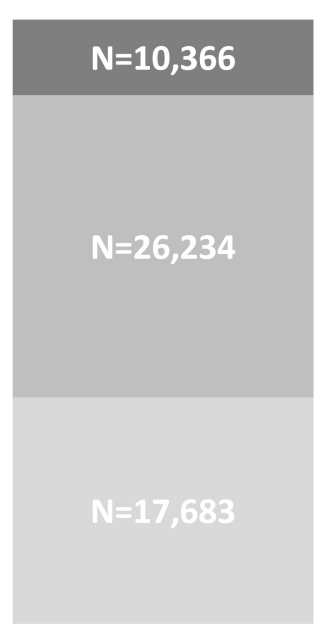

CLD of other causes

Alcohol-related CLD

ijcp_13841_f2.jpg

This article is protected by copyright. All rights reserved 

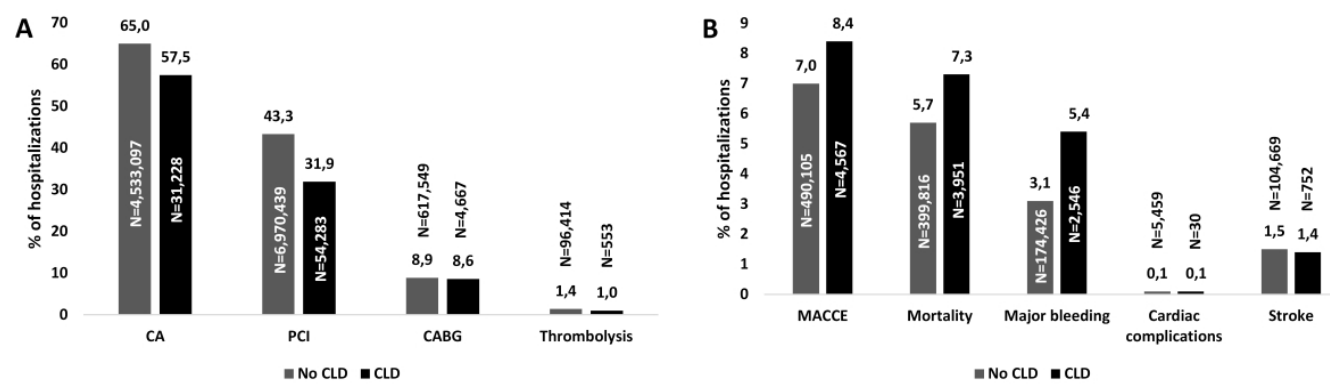

ijcp_13841_f3.jpg 


\section{$\operatorname{aOR}(95 \% \mathrm{Cl})$}

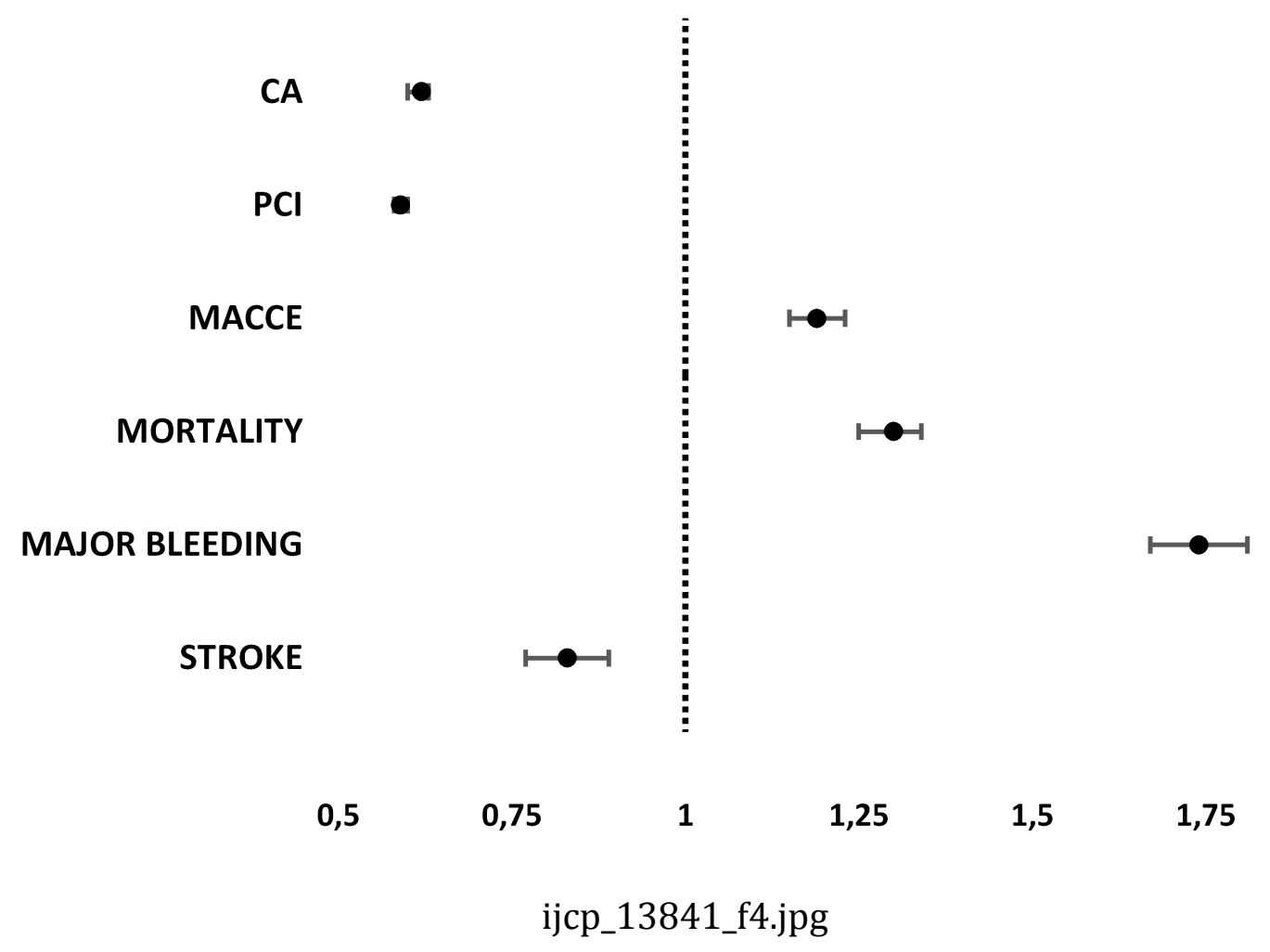

This article is protected by copyright. All rights reserved 


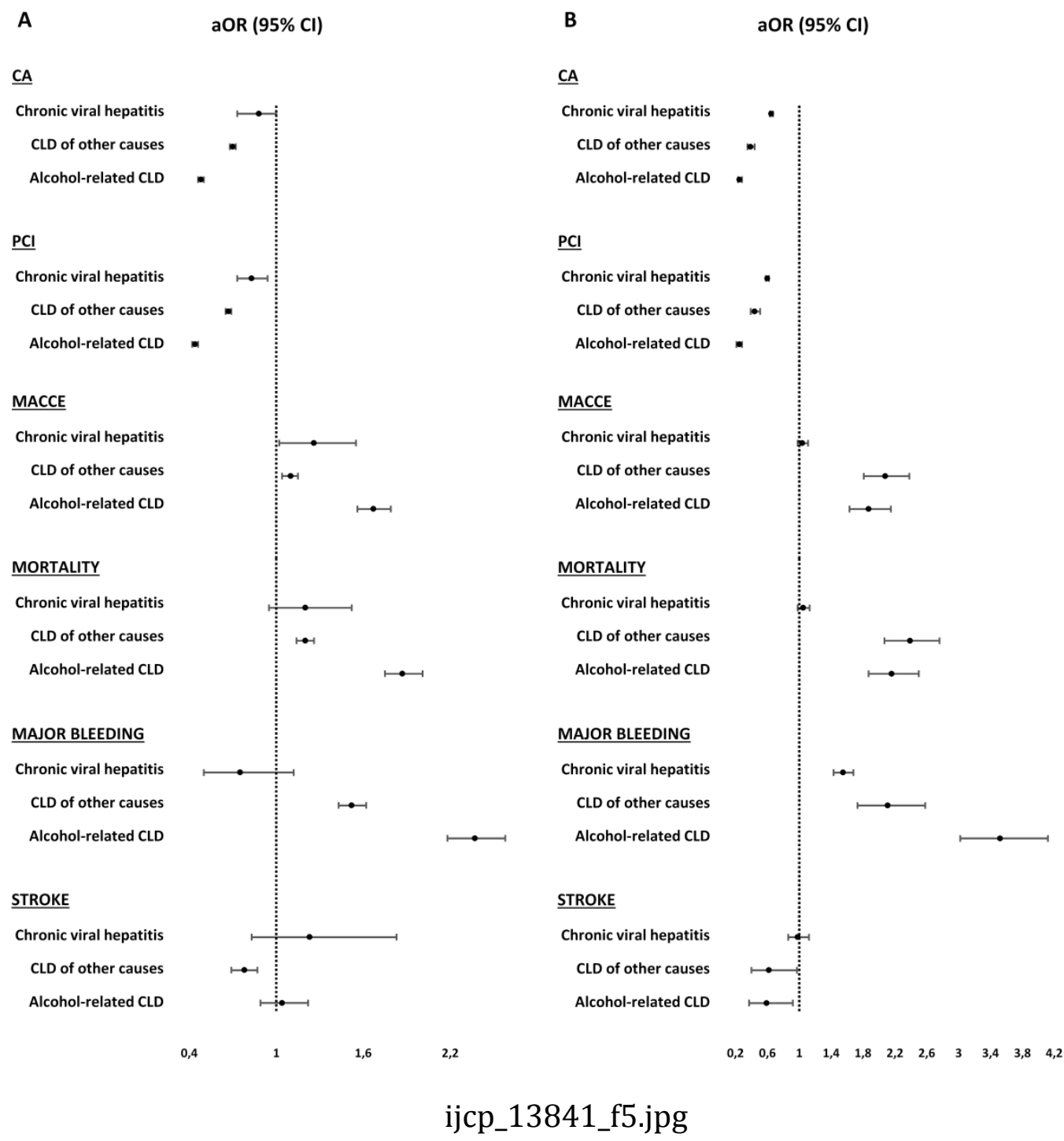

This article is protected by copyright. All rights reserved 


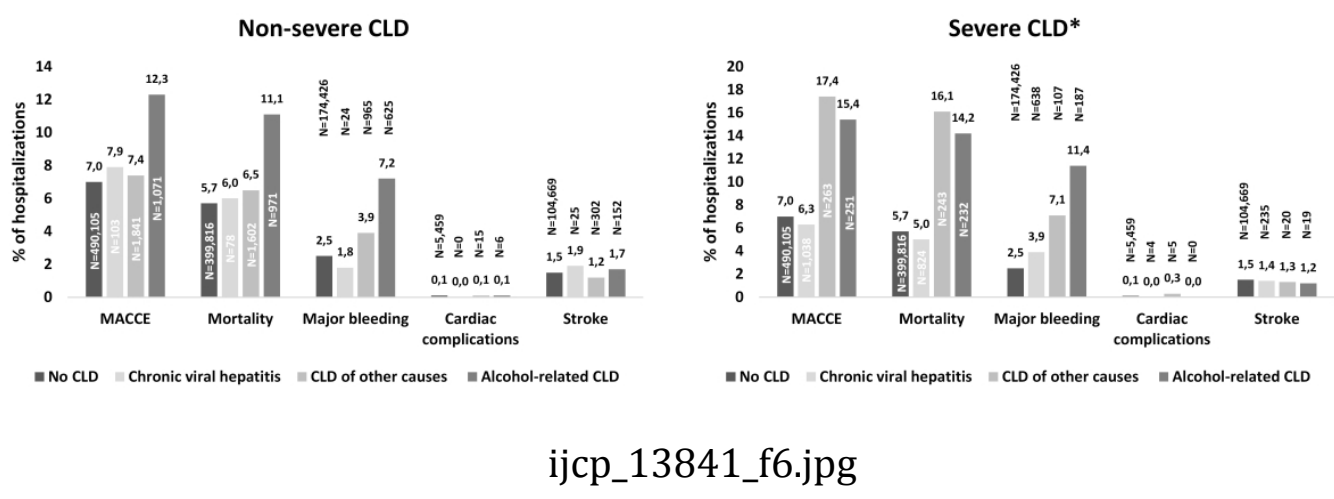

This article is protected by copyright. All rights reserved 